Journal of Computer Science 5 (1): 71-78, 2009

ISSN 1549-3636

(C) 2009 Science Publications

\title{
Error Free Iterative Morphological Decomposition Algorithm for Shape Representation
}

\author{
${ }^{1}$ V. Vijaya Kumar, ${ }^{2}$ A. Srikrishna and ${ }^{3}$ G. Hemantha Kumar \\ ${ }^{1}$ Department of Computer Science and Engineering and Information Technology, \\ Godavari Institute of Engineering and Technology, Rajahmundry, India \\ ${ }^{2}$ Department of Computer Science and Engineering, Rayapati Venkat Ranga Rao and Jagarlamudi, \\ Chandramouli College of Engineering Guntur, India \\ ${ }^{3}$ Department of Studies in Computer Science, University of Mysore, Karnataka, India
}

\begin{abstract}
Problem statement: A generalized skeleton transform allows a shape to be represented as a collection of modestly overlapped octagonal shape parts. One problem with several generalized Morphological skeleton transforms is that they generate noise after decomposition. The noise rate may not be effective for ordinary images; however this effect will be more when applied on printed or handwritten characters. Approach: The present study tackled this issue by applying a noise removal algorithm after morphological decomposition. Results: The algorithm was applied on various types of decomposition images. The Present method was compared with generalized skeleton algorithm and octagon-generating decomposition algorithm. Conclusion: The error rates with original image were evaluated using various error functions. The experimental results indicated that the present decomposition algorithm produces images with good clarity when compared with other algorithms.
\end{abstract}

Key words: Shape, noise, printed hand written characters, error-rate, error functions

\section{INTRODUCTION}

SHAPE representation is an important issue in image processing and computer vision. Efficient shape representation provides the foundation for the development of efficient algorithms for many shaperelated processing tasks, such as image coding ${ }^{[1,2]}$, shape matching and object recognition ${ }^{[3-7]}$, contentbased video processing ${ }^{[8,9]}$ and image data retrieval $^{[10,11]}$. Mathematical morphology is a shapebased approach to image processing ${ }^{[12,13]}$. Basic morphological operations can be given interpretations using geometric terms of shape, size and distance. Therefore, mathematical morphology is especially suited for handling shape-related processing and operations. Mathematical morphology also has a welldeveloped mathematical structure, which facilitates the development and analysis of morphological image processing algorithms. A number of morphological shape representation schemes have been proposed $^{[1,2,14-28,30]}$. Many of them use the structural approach. That is, a given shape is described in terms of its simpler shape components and the relationships among the components.
The notion of skeleton or medial axis transform was first introduced by Blum ${ }^{[34]}$. Lantuejoul showed that the skeleton can be computed using morphological operations ${ }^{[35]}$. The term 'Skeleton' is often used to describe thinning algorithms that preserve homotopy but do not necessarily support exact shape reconstruction ${ }^{[36,37]}$. In this study, our focus is on building efficient structural shape representations that allow exact as well as approximate reconstructions of the input shapes. Therefore, we are following a structural and algebraic approach to shape representation. Recently new algorithms for skeletonization and thinning, for 2D images based on primitive concept approach were proposed ${ }^{[31-33]}$.

The 'Morphological Skeleton Transform' (MST) is a leading morphological shape representation $\operatorname{algorithm}^{[14]}$. In the MST, a given shape is represented as a union of all maximal disks contained in the shape. In general, there is much overlapping among the maximal disks. The 'Morphological Shape Decomposition' (MSD) is another important morphological shape representation scheme ${ }^{[15]}$, in which a given shape is represented as a union of certain

Corresponding Author: V. Vijaya Kumar, Department of Computer Science and Engineering and Information Technology, Godavari Institute of Engineering and Technology, Rajahmundry, India 
disks contained in the shape. The overlapping among representative disks of different sizes is eliminated. A new morphological shape representation algorithm that can be viewed as a compromise between the MST and the MSD was recently proposed ${ }^{[23,29]}$. In this scheme, overlapping among representative disks of different sizes is allowed, but severe overlapping among such disks is avoided. This algorithm is called as 'Overlapped Morphological Shape Decomposition' (OMSD). The advantages of these basic algorithms include that they have simple and well-defined mathematical characterizations and they are easy and efficient to implement. This study focuses on building efficient structural shape representations that allow exact as well as approximate reconstructions of the input shapes.

\section{MATERIALS AND METHODS}

In this study, we will first introduce an algorithm for generating skeleton points which will find a special maximal octagon for each image point of a given shape. In the decomposition algorithm a given shape will be decomposed into a collection of modestly overlapping disk components. And it will also provide enough information to allow efficient collection of disk components to be selected for exact representation of the given shape. The exact representation of the given shape is achieved through a noise removal algorithm.

An algorithm for representation of skeleton points of a given image in the form of flow chart is shown in Fig. 1. In this algorithm, the skeleton points are derived by repeatedly applying erosion operation using eight structuring elements in the following order: $\mathrm{B}_{0}, \mathrm{~B}_{1}, \ldots \mathrm{B}_{7}, \mathrm{~B}_{0}, \mathrm{~B}_{1}, \ldots \mathrm{B}_{7}, \mathrm{~B}_{0}, \mathrm{~B}_{1}, \ldots$ as shown in Fig. 2 The symbols ' $*$ ', ,', ' + ' represents origin, zero and one respectively. That is these eight structuring elements will be applied in cyclic sequence. This process of representation of skeleton points is same as 'octagongenerating decomposition algorithm.

The proposed (EFMD) Error Free Morphological Decomposition algorithm, while reconstructing the image removes noise and this is given in the form of a flow chart in Fig. 3 The proposed algorithm utilizes the number of skeleton points, in their co-ordinate positions, corresponding structuring elements and noise removal filter for reconstruction of the image. The process will be repeated for the skeleton points obtained by the algorithm to generate skeleton points shown in Fig. 1.

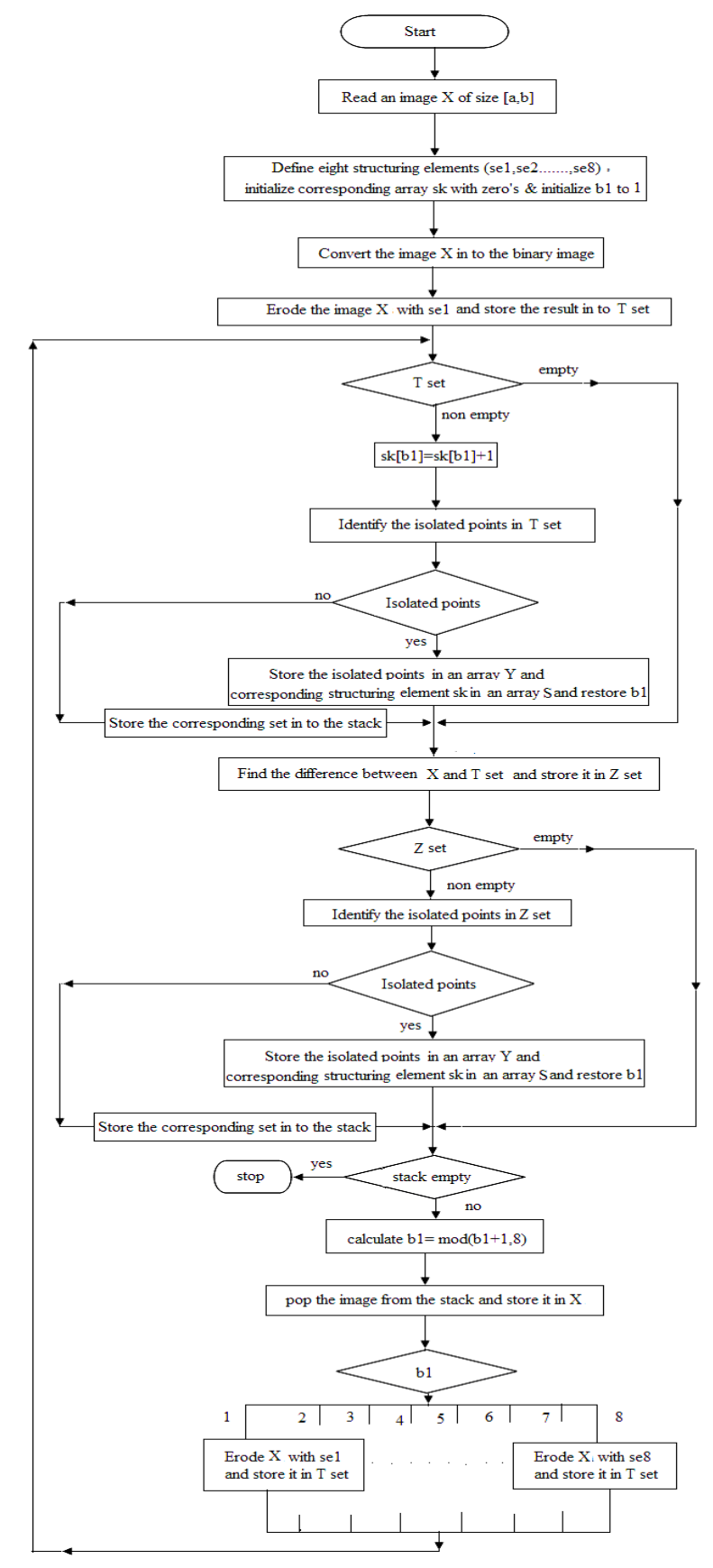

Fig. 1: Flowchart for algorithm to generate skeleton points

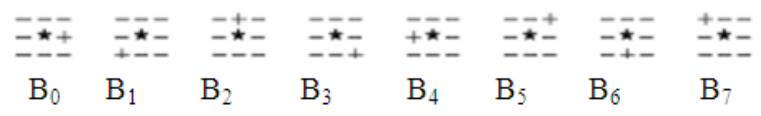

Fig. 2: Eight two-point structuring elements 


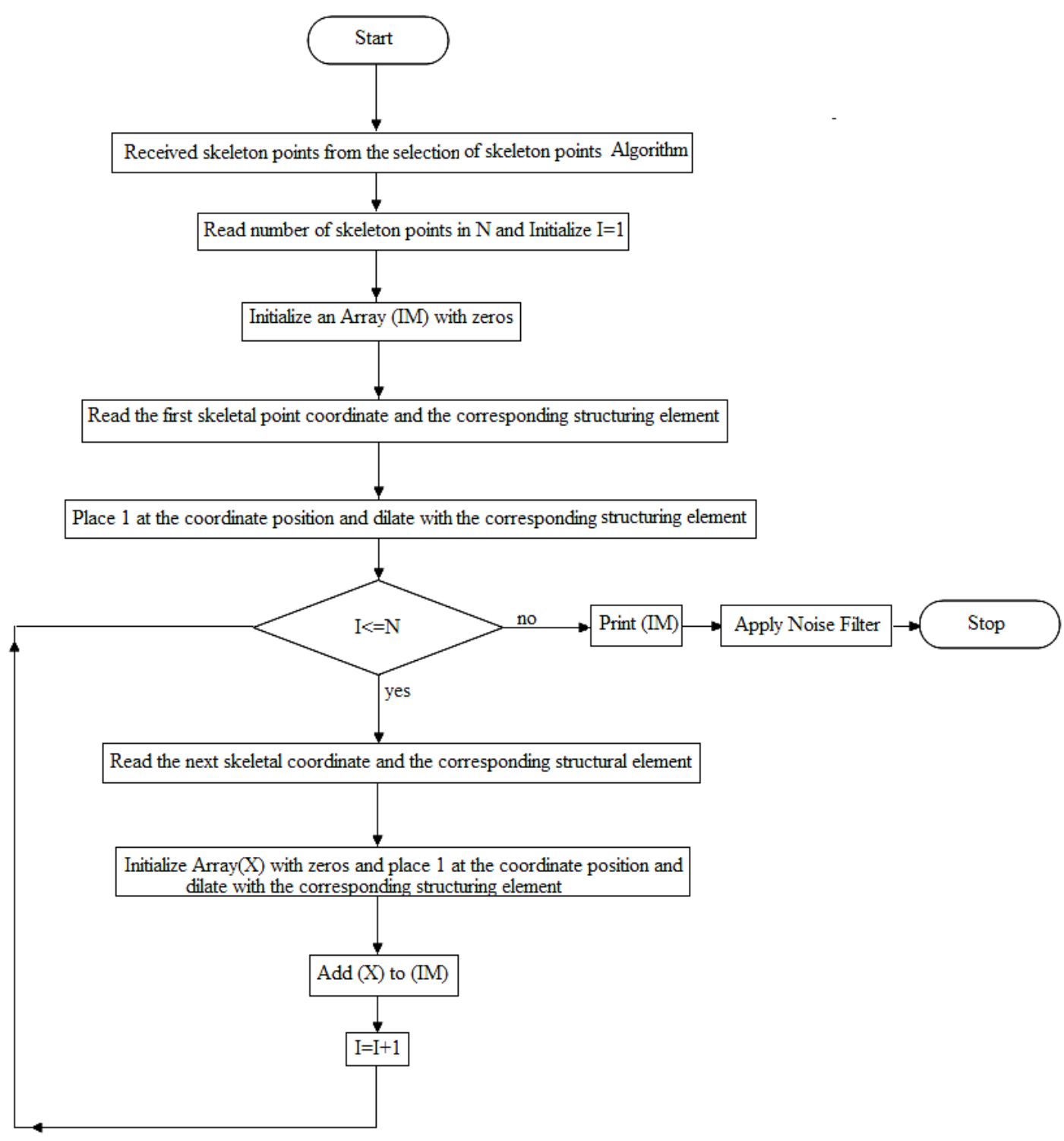

Fig. 3: Flowchart for noise removal for reconstruction of image

\section{RESULTS}

To test the integrity of the noise removal decomposition algorithm nine different images are taken and they are showed in the Fig. 4. Figure 5-7 show the reconstructed images using (GST) Generalized Skeleton Transform algorithm, (OGD) Octagon-Generating Decomposition algorithm and (EFMD) Error Free Morphological Decomposition algorithm. Various error functions as stated in equation (1-7) are applied on all reconstructed images using the three algorithms. The error rate is defined as the ratio between the number of image points that are not represented and the number of points in the original shape.

Error functions:

AEPP : Average error per pixel

MSE : Mean square error

RMSE : Root mean square error

SNR (ms) : Signal to noise ratio (mean square)

SNR (rms) : Signal to noise ratio (root mean square)

PSNR : Peak signal to noise ratio

Error-Rate : Error-rate per pixel 


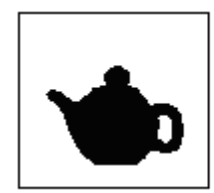

(a)

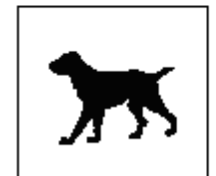

(d)

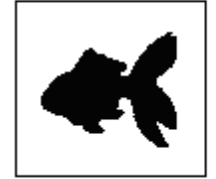

(g)

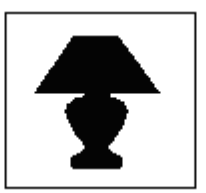

(b)

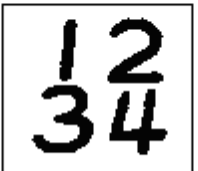

(e)

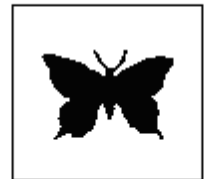

(h)

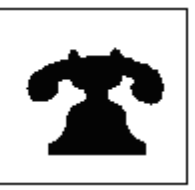

(c)

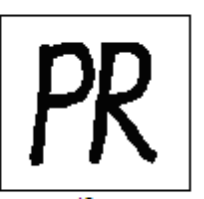

(f)

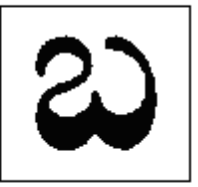

(i)

Fig. 4: Shape images used in the experiments. (a): Teapot; (b): Lamp; (c): Telephone; (d): Dog; (e): Digits; (f): Letters; (g): Fish; (h): Butterfly; (i): Telugu character

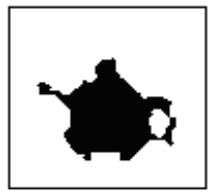

(a)

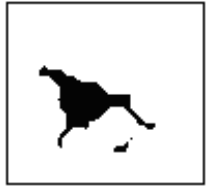

(d)

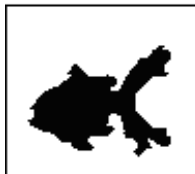

(g)

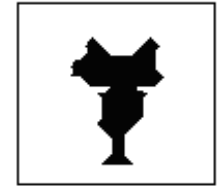

(b)

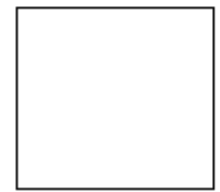

(e)

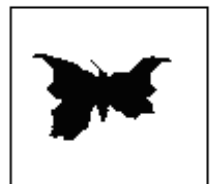

(h)

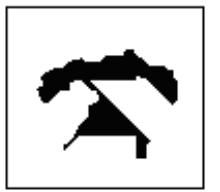

(c)

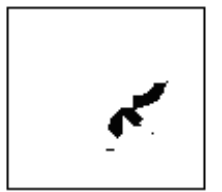

(f)

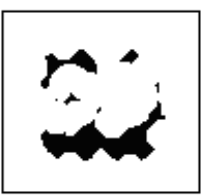

(1)

Fig. 5: Shape images after reconstruction using GST algorithm. (a): Teapot; (b): Lamp; (c): Telephone; (d): Dog (e): Digits; (f): Letters; (g): Fish; (h): Butterfly; (i): Telugu character

AEPP $=\frac{1}{M \times N} \sum_{i=0}^{M-1} \sum_{j=0}^{N-1}|f(x, y)-g(x, y)|$

$$
\text { MSE }=\frac{1}{M \times N} \sum_{i=0}^{M-1} \sum_{j=0}^{N-1}(f(x, y)-g(x, y))^{2}
$$

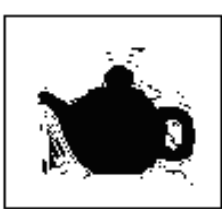

(a)

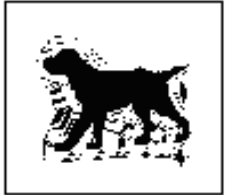

(d)

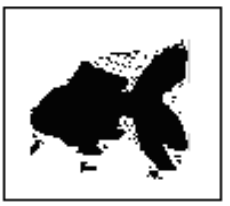

(g)

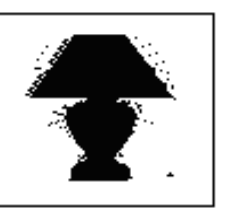

(b)

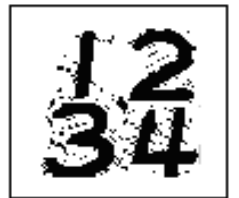

(e)

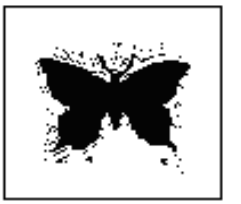

(h)

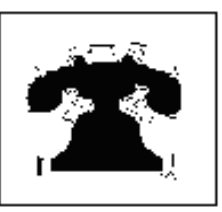

(c)

(f)

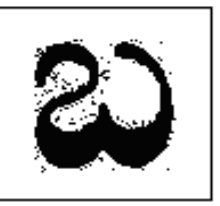

(i)

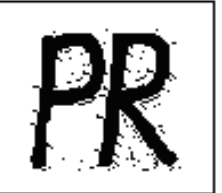

Fig. 6: Shape images after reconstruction using OGD algorithm. (a): Teapot; (b): Lamp; (c): Telephone; (d): Dog (e): Digits; (f): Letters; (g): Fish; (h): Butterfly; (i): Telugu character

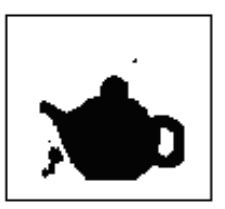

(a)

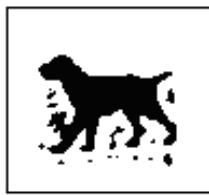

(d)

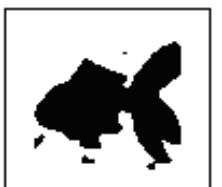

(g)

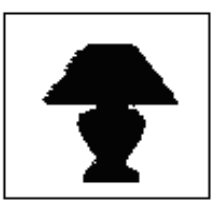

(b)

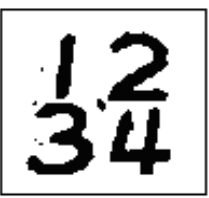

(e)

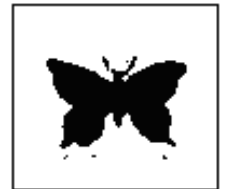

(h)

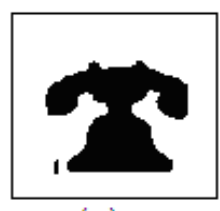

(c)

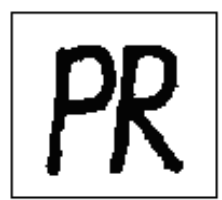

(f)

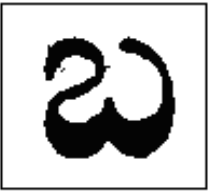

(i)
Fig. 7: Shape images after reconstruction using EFMD algorithm. (a): Teapot; (b): Lamp; (c): Telephone; (d): Dog; (e): Digits; (f): Letters; (g): Fish; (h): Butterfly; (i): Telugu character

$$
\text { RMSE }=\sqrt{\frac{1}{M \times N} \sum_{i=0}^{M-1} \sum_{j=0}^{N-1}(f(x, y)-g(x, y))^{2}}
$$




$$
\operatorname{SNR}(\mathrm{ms})=\frac{\sum_{\mathrm{i}=0}^{M-1} \sum_{\mathrm{j}=0}^{N-1} \mathrm{~g}(\mathrm{x}, \mathrm{y})^{2}}{\sum_{\mathrm{i}=0}^{\mathrm{M}-1} \sum_{\mathrm{j}=0}^{\mathrm{N}-1}(\mathrm{~g}(\mathrm{x}, \mathrm{y})-\mathrm{f}(\mathrm{x}, \mathrm{y}))^{2}}
$$

$$
\operatorname{SNR}(\mathrm{rms})=\sqrt{\frac{\sum_{\mathrm{i}=0}^{M-1} \sum_{\mathrm{j}=0}^{N-1} \mathrm{~g}(\mathrm{x}, \mathrm{y})^{2}}{\sum_{\mathrm{i}=0}^{M-1} \sum_{\mathrm{j}=0}^{\mathrm{N}-1}(\mathrm{~g}(\mathrm{x}, \mathrm{y})-\mathrm{f}(\mathrm{x}, \mathrm{y}))^{2}}}
$$

$\mathrm{PSNR}=10 * \log _{10}\left(\frac{255^{2}}{\mathrm{MSE}}\right)$

$$
\text { Error_Rate }=\frac{\sum_{i=0}^{M-1} \sum_{j=0}^{N-1}|f(x, y)-g(x, y)|}{\sum_{i=0}^{M-1} \sum_{j=0}^{N-1} f(x, y)}
$$

Let:

$\mathrm{f}(\mathrm{x}, \mathrm{y}) \quad=$ Represent an input image

$\mathrm{g}(\mathrm{x}, \mathrm{y})=$ Represent reconstructed image

$\mathrm{M}$ and $\mathrm{N}=$ The sizes of input image and reconstructed image

A closely related objective measure is MSE. RMSE, SNR(ms), SNR(rms), PSNR are the defacto standards used in the image processing community. It is so commonly used for three reasons 1 ) because some objective measure is needed; (2) because it is possible to relate MSE to theoretical issues related to rate/distortion curves and least-squares minimization in statistical theory more easily than with any other measures and (3) because PSNR is a logarithmic measure which correlates with the logarithmic response to image intensity of the HVS. Generally speaking, as a rule of thumb, the higher PSNR will frequently correspond to better decompression noticeably. But the present study has tested this for reconstruction of the images.

\section{DISCUSSION}

All the error functions reflect this fact between the original image $f(x, y)$ and the reconstructed image $g(x, y)$. Table 1-3 show the error rates of reconstructed images with original image using GST algorithm, OGD algorithm and the EFMD algorithm, respectively. Table 1-3 average error rate of error functions on all nine different images is evaluated. It is evident that the error rate of the present method is reduced to half or even more when compared with other two algorithms.

One more interesting point is that the error rate of the proposed method is less than the other two methods for all images by using all error functions. The PSNR is high for the present method for all images. It indicates that it has high signal to noise ratio. Figure 8-10 show reconstructed images after reversing the background color of the image for generalized skeleton algorithm, octagon generating decomposition algorithm and, proposed error free decomposition algorithm respectively. Even in this case the proposed error free reconstruction algorithm has shown less noise when compared with other algorithms. The above fact is evident from the Table 4-6. When we reverse the background of the image, the shape component is not clear with GST, due to the effect of dilation on background intensity as shown in Fig. 5. By this, error rate is increased.

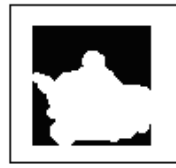

(a)

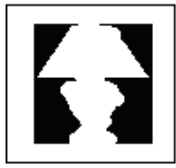

(b)

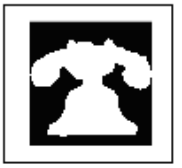

(c)

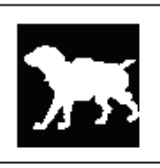

(d)
Fig. 8: Shape images after reconstruction using GST algorithm with image and background inverted. (a): Teapot; (b): Lamp; (c): Telephone; (d): Dog

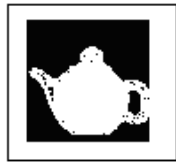

(a)

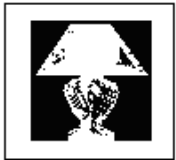

(b)

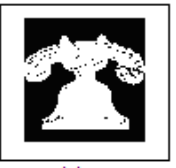

(c)

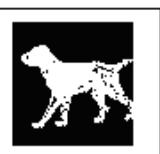

(d)
Fig. 9: Shape images after reconstruction using OGD algorithm with image and background inverted: (a): Teapot; (b): Lamp; (c): Telephone; (d): Dog

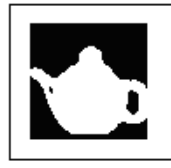

(a)

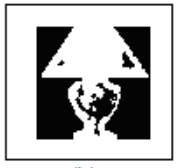

(b)

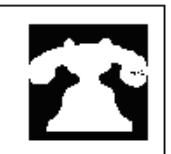

(c)

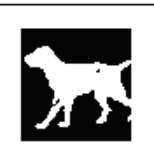

(d)
Fig. 10: Shape images after reconstruction and using EFMD algorithm with image and background inverted (a): Teapot; (b): Lamp; (c): Telephone; (d): Dog 
J. Computer Sci., 5 (1): 71-78, 2009

Table 1: Error calculations using different error functions after reconstruction using generalized skeleton transform algorithm

\begin{tabular}{llllllll}
\hline & AEPP & MSE & RMSE & SNR (ms) & SNR (rms) & PSNR & Error-rate \\
\hline Teapot & 0.061020000 & 0.061020000 & 0.24702000 & 11.04700 & 3.3237000 & 60.276000 & 9.953400 \\
Lamp & 0.180820000 & 0.180820000 & 0.42523000 & 4.130900 & 2.0325000 & 55.558000 & 31.93900 \\
Telephone & 0.170610000 & 0.170610000 & 0.41305000 & 4.367200 & 2.0898000 & 55.811000 & 29.69800 \\
Dog & 0.133270000 & 0.133270000 & 0.36506000 & 6.494600 & 2.5485000 & 56.884000 & 18.20000 \\
Digits & & & & & & \\
Letters & 0.280820000 & 0.280820000 & 0.52992000 & 3.389500 & 1.8411000 & 53.647000 & 41.84900 \\
Fish & 0.071020000 & 0.071020000 & 0.26650000 & 9.069000 & 3.0115000 & 59.617000 & 12.39300 \\
Butterfly & 0.077755000 & 0.077755000 & 0.27885000 & 9.343800 & 3.0568000 & 59.224000 & 11.98500 \\
Telugu character & 0.197750000 & 0.197750000 & 0.44470000 & 3.950600 & 1.9876000 & 55.170000 & 33.89100 \\
Average & 0.146633125 & 0.146633125 & 0.37129125 & 6.474075 & 2.4864375 & 57.023375 & 23.73855 \\
\hline
\end{tabular}

Table 2: Error calculations using different error functions after reconstruction using octagon-generating decomposition algorithm

\begin{tabular}{|c|c|c|c|c|c|c|c|}
\hline & AEPP & MSE & RMSE & SNR (ms) & SNR (rms) & PSNR & Error-rate \\
\hline Teapot & 0.04286 & 0.04286 & 0.20702 & 13.32400 & 3.65020 & 61.81100 & 6.99070 \\
\hline Lamp & 0.02633 & 0.02633 & 0.16225 & 20.50400 & 4.52810 & 63.92700 & 4.65030 \\
\hline Telephone & 0.02980 & 0.02980 & 0.17261 & 18.28100 & 4.27560 & 63.38900 & 5.18650 \\
\hline Dog & 0.08490 & 0.08490 & 0.29137 & 7.62500 & 2.76130 & 58.84200 & 11.59400 \\
\hline Digits & 0.06347 & 0.06347 & 0.25193 & 9.93890 & 3.15260 & 60.10500 & 9.14170 \\
\hline Letters & 0.04000 & 0.04000 & 0.20000 & 15.86700 & 3.98340 & 62.11000 & 5.96110 \\
\hline Fish & 0.03755 & 0.03755 & 0.19378 & 14.26100 & 3.77640 & 62.38500 & 6.55270 \\
\hline Butterfly & 0.03837 & 0.03837 & 0.19588 & 15.91000 & 3.98870 & 62.29100 & 5.91380 \\
\hline Telugu character & 0.04028 & 0.04028 & 0.20071 & 13.48500 & 3.67220 & 62.08000 & 6.90380 \\
\hline Average & 0.04484 & 0.04484 & 0.20839 & 14.35510 & 3.75428 & 61.88222 & 6.98829 \\
\hline
\end{tabular}

Table 3: Error calculations using different error functions after reconstruction using error free decomposition algorithm

\begin{tabular}{llllllll}
\hline & AEPP & MSE & RMSE & SNR $(\mathrm{ms})$ & SNR $(\mathrm{rms})$ & PSNR & Error-rate \\
\hline Teapot & 0.02531 & 0.02531 & 0.15908 & 21.31500 & 4.61680 & 64.09900 & 4.12780 \\
Lamp & 0.01531 & 0.01531 & 0.12372 & 32.89300 & 5.73530 & 66.28200 & 2.70370 \\
Telephone & 0.01429 & 0.01429 & 0.11952 & 35.94300 & 5.99520 & 66.58200 & 2.48670 \\
Dog & 0.05000 & 0.05000 & 0.22361 & 12.66100 & 3.55830 & 61.14100 & 6.82830 \\
Digits & 0.02878 & 0.02878 & 0.16963 & 21.59600 & 4.64710 & 63.54100 & 4.14460 \\
Letters & 0.01857 & 0.01857 & 0.13628 & 32.62600 & 5.71200 & 65.44200 & 2.76760 \\
Fish & 0.02143 & 0.02143 & 0.14639 & 23.58100 & 4.85600 & 64.82100 & 3.73930 \\
Butterfly & 0.01653 & 0.01653 & 0.12857 & 35.50600 & 5.95870 & 65.94800 & 2.54800 \\
Telugu character & 0.02075 & 0.02075 & 0.14406 & 24.81200 & 4.98110 & 64.96000 & 3.55650 \\
Average & 0.02344 & 0.02344 & 0.15010 & 26.77033 & 5.11783 & 64.75733 & 3.65583 \\
\hline
\end{tabular}

Table 4: Error calculations using different error functions after reconstruction using generalized skeleton transform algorithm (image and background inverted)

\begin{tabular}{llccrrrr}
\hline & AEPP & MSE & RMSE & SNR $(\mathrm{ms})$ & SNR $(\mathrm{rms})$ & PSNR & Error-Rate \\
\hline Teapot & 0.07000 & 0.07000 & 0.26458 & 6.52770 & 2.55490 & 59.68000 & 18.09100 \\
Lamp & 0.02000 & 0.02000 & 0.14142 & 22.69400 & 4.76380 & 65.12100 & 4.60960 \\
Telephone & 0.01122 & 0.01122 & 0.10595 & 38.90900 & 6.23770 & 67.62900 & 2.63790 \\
Dog & 0.03490 & 0.03490 & 0.18681 & 8.67250 & 2.94490 & 62.70300 & 13.03400 \\
Average & 0.03403 & 0.03403 & 0.17469 & 19.20080 & 4.12533 & 63.78325 & 9.59313 \\
\hline
\end{tabular}

Table 5: Error calculations using different error functions after reconstruction using octagon-generating decomposition algorithm (image and background inverted)

\begin{tabular}{llllrlrr}
\hline & AEPP & MSE & RMSE & SNR (ms) & SNR (rms) & PSNR & Error-Rate \\
\hline Teapot & 0.01122 & 0.01122 & 0.10595 & 33.72700 & 5.80750 & 67.62900 & 2.90080 \\
Lamp & 0.07939 & 0.07939 & 0.28176 & 4.46530 & 2.11310 & 59.13300 & 18.29700 \\
Telephone & 0.02225 & 0.02225 & 0.14915 & 18.12800 & 4.25780 & 64.65800 & 5.22780 \\
Dog & 0.02449 & 0.02449 & 0.15649 & 9.93330 & 3.15170 & 64.24100 & 9.14630 \\
Average & 0.03434 & 0.03434 & 0.17334 & 16.56340 & 3.83253 & 63.91525 & 8.89298 \\
\hline
\end{tabular}

Table 6: Error calculations using different error functions after reconstruction using error free decomposition algorithm.(image and background

\begin{tabular}{llllrrrr}
\multicolumn{2}{c}{ inverted) } & & & & & \\
\hline & AEPP & MSE & RMSE & SNR (ms) & SNR (rms) & PSNR & Error-Rate \\
\hline Teapot & 0.00714 & 0.00714 & 0.08452 & 54.40000 & 7.37560 & 69.59200 & 1.84600 \\
Lamp & 0.06694 & 0.06694 & 0.25873 & 5.45730 & 2.33610 & 59.87400 & 15.42800 \\
Telephone & 0.00898 & 0.00898 & 0.09476 & 47.59100 & 6.89860 & 68.59800 & 2.11030 \\
Dog & 0.01714 & 0.01714 & 0.13093 & 15.10700 & 3.88680 & 65.79000 & 6.40240 \\
Average & 0.02505 & 0.02505 & 0.14223 & 30.63883 & 5.12428 & 65.96350 & 6.44668 \\
\hline
\end{tabular}




\section{CONCLUSION}

In this study, we have introduced an error free decomposition algorithm. By this, noise rate is reduced. The proposed noiseless decomposition algorithm is more efficient than generalized skeleton algorithm or octagon-generating decomposition algorithm. The experimental results using seven error functions on nine images show that the new error free decomposition algorithm produces a more clarity-based shape representation than the other two algorithms.

\section{ACKNOWLEDGEMENT}

The researchers would like to express their gratitude to Sri K.V.V. Satya Narayana Raju, Chairman and K. Sashi Kiran Varma, Managing Director, Chaitanya group of Institutions for providing necessary infrastructure. And they would like to thank Dr.G.V.S.Ananta Lakshmi for her invaluable suggestions and constant encouragement that led to improvise the presentation quality of this study.

\section{REFERENCES}

1. Hasan, Y.M.Y. and L.J. Karam, 2000. Morphological reversible contour representation. IEEE Trans. Patt. Anal. Mach. Intell., 22: 227-240. http://www2.computer.org/portal/web/csdl/doi/10.1 109/34.841755

2. Kresch, R. and D. Malah, 1998. Skeleton-based morphological coding of binary images. IEEE Trans. Image Process., 7: 1387-1399. ieeexplore.ieee.org/iel4/83/15529/00718480.pdf?ar number $=718480$

3. Pitas, I. and A. Maglara, 1991. Range image analysis using morphological signal decomposition. Patt. Recog., 24: 165-181. DOI: 10.1016/0031-3203(91)90086-K

4. Trahanias, P.E., 1992. Binary shape recognition using the morphological skeleton transform. Patt. Recog., 25: 1277-1288. DOI: 10.1016/00313203(92)90141-5

5. Ei-Kwae, E.A. and M.R. Kabuka, 2000. Binary object representation and recognition using the Hilbert morphological skeleton transform. Patt. Recog., 33: 1621-1636. DOI: 10.1016/S00313203(99)00169-7

6. Belongie, S., J. Malik and J. Puzicha, 2002. Shape matching and object recognition using shape contexts. IEEE Trans. Patt. Anal. Mach. Intell., 24: 509-522. DOI: $10.1109 / 34.993558$
7. Siddiqi, K., A. Shokoufandeh, S.J. Dickinson and S.W. Zucker, 1999.Shock graphs and shape matching. Int. J. Comput. Vis., 35: 13-32. DOI: 10.1023/A:1008102926703

8. Salembier, P., P. Brigger, J.R. Casas and M. Pardas, 1996. Morphological operators for image and video compression. IEEE Trans. Image Process., 5: 881-897. http://gpstsc.upc.es/imatge/_Montse/publication_data.html

9. Jasinschi, R.S. and J.M.F. Moura, 1995. Contentbased video sequence representation., Proceeding of the IEEE International Conference Image Processing, Oct. 23-26, IEEE Computer Society, Washington, DC., USA., pp: 2229. http://portal.acm.org/citation.cfm?id=841149\&dl= GUIDE\& coll $=$ GUIDE $\&$ CFID $=26503854 \&$ CFTO $\mathrm{KEN}=73362793$

10. Sharvit, D., J. Chan, H. Tek and B. Kimia, 1998. Symmetry-based indexing of image databases. J. Vis. Commun. Image Represent., 9: 366-380. http://www.lems.brown.edu/ des/papers/symmetry _based_indexing-CVPR98.ps.gz

11. Latecki, L.J. and R. Lakamper, 2002. Application of planar shape comparison to object retrieval in image databases. Patt. Recog., 35: 15-29. DOI: 10.1016/S0031-3203(01)00039-5

12. Serra, J., 1983. Image Analysis and Mathematical Morphology. Academic, London, UK., ISBN: 10: 0126372403, pp: 610.

13. Haralick, R.M., S.R. Sternberg and X. Zhuang, 1987. Image analysis using mathematical morphology. IEEE Trans. Patt. Anal. Mach. Intell., 9: 532-550. http://portal.acm.org/ citation.cfm?id=28802

14. Maragos, P.A. and R.W. Schafer, 1986. Morphological skeleton representation and coding of binary images. IEEE Trans. Acoust. Speech Signal Process., 34: 1228-1244. http://ieeexplore.ieee.org/xpl/freeabs_all.jsp?arnum ber $=1164959$

15. Pitas, I. and A.N. Venetsanopoulos, 1990. Morphological shape decomposition. IEEE Trans. Pattern Anal. Mach. Intell., 12: 38-45. DOI: 10.1109/34.41382

16. Reinhardt, J.M. and W.E. Higgins, 1996. Comparison between the morphological skeleton and morphological shape decomposition. IEEE Trans. Patt. Anal. Mach. Intell., 18: 951-957. DOI: $10.1109 / 34.537351$ 
17. Maragos, P., 1988. Morphology-based symbolic image modeling multi-scale nonlinear smoothing and pattern spectrum. Proceedings of the Conference on Computer Vision Pattern Recognition, June 5-9, IEEE Computer Society, Washington DC., USA., pp: 766-773. DOI: 10.1109/CVPR.1988.196321

18. Pitas, I. and A.N. Venetsanopoulos, 1992. Morphological shape representation. Patt. Recog., 25: 555-565. DOI: 10.1016/0031-3203(92)90073-R

19. Reinhardt, J.M. and W.E. Higgins, 1996. Efficient morphological shape representation. IEEE Trans. Image Process., 5: 89-101.

http://ieeexplore.ieee.org/search/wrapper.jsp?arnu mber $=481673$

20. Xu, J., 1996. Morphological decomposition of 2-D binary shapes into conditionally maximal convex polygons. Patt. Recog., 29: 1075-1104. http://ieeexplore.ieee.org/search/wrapper.jsp?arnu mber $=413538$

21. Xu, J., 2001. Morphological representation of 2-D binary shapes using rectangular components. Patt. Recog., 34: 277-286. DOI: 10.1109/ICIP.1999.823020

22. Xu, J., 2001. Morphological decomposition of 2-D binary shapes into convex polygons: A heuristic algorithm. IEEE Trans. Image Process., 10: 61-71. DOI: $10.1109 / 83.892443$

23. $\mathrm{Xu}$, J., 2001. Efficient morphological shape representation with overlapping disk components. IEEE Trans. Image Process., 10: 1346-1356. DOI: 10.1109/83.941858

24. Xu, J., 2003. A generalized discrete morphological skeleton transform with multiple structuring elements for the extraction of structural shape components. IEEE Trans. Image Process., 12: 1677-1686. DOI: 10.1109/TIP.2003.819225

25. $\mathrm{Xu}, \mathrm{J} ., 2003$. Efficient morphological shape representation by varying overlapping levels among representative disks. Patt. Recog., 36: 429-437. DOI: 10.1016/S0031-3203(02)00075-4

26. Held, A. and K. Abe, 1994. On the decomposition of binary shapes into meaningful parts. Patt. Recog., 27: 637-647. DOI: 10.1016/00313203(94)90043-4.

27. Ronse, C. and B. Macq, 1991. Morphological shape and region description. Signal Process., 25: 91-106. DOI: 10.1016/0165-1684(91)90041-G

28. Goutsias, J. and D. Schonfeld, 1991. Morphological representation of discrete and binary images. IEEE Trans. Signal Process., 39: 1369-1379. DOI: 10.1109/78.136543
29. Xu, J., 1998. Efficient morphological shape representation without searching. Proceedings of the IEEE International Conference on Image Processing, Oct. 4-7, IEEE Computer Society, Washington DC., USA., pp: 262-266. DOI: 10.1109/ICIP.1998.723360

30. Xu, J., 2007. Morphological decomposition of 2-D binary shapes into modestly overlapped octagonal and disk components. IEEE Trans. Image Process., 16: 337-348. DOI: 10.1109/TIP.2006.888328

31. Vijaya, V. and Kumar et al., 2008. An improved iterative morphological decomposition approach for image skeletonization. GVIP. J., 8: 47-54. www.icgst.com/gvip/Volume8/Issue1/P115081200 4.pdf

32. Vijaya, V. and Kumar et al., 2008. A new skeletonization method based on connected component approach. Int. J. Comput. Sci. Network Sec., 8: 133-137. paper.ijcsns.org/07_book/200802/20080218.pdf

33. Vijaya Kumar, V. et al., 2006. A comparison on morphological skeleton transform with multiple structuring elements.pg.no-8. Proceedings of the International Conference on ICORG, June 6-8, NIRD Campus, Hyderabad. www.icorg.org/html/ICORG-Technical- program.pdf

34. Blum, H., 1967. A Transformation for Extracting New Descriptors of Shape. In: Models for the Perception of Speech and Visual Forms, Wathen-Dunn, W. (Ed.). MIT Press, Cambridge, MA., pp: 362-380.

35. Lantuejoul, C., 1980. Skeletonization in Quantitative Metallography. In: Issues of Digital Image Processing, Haralick, R.M. and J.C. Simon (Eds.). Sijthoff and Noordhoff, Groningen, Netherlands. DOI: 10.110910.1109/34.56190

36. Pudney, C., 1998. Distance-ordered homotopic thinning: A skeletonization algorithm for 3D digital images. Comput. Vis. Image Understand, 72: 404-413. DOI: 10.1006/cviu.1998.0680

37. Ranwez, V. and P. Soille, 2002. Order independent homotopic thinning for binary and grey tone anchored skeletons. Patt. Recog. Lett., 23: 687-702. DOI: 10.1016/S0167-8655(01)00146-5 